\title{
NEUROMYELITIS OPTIC SPECTRUM DISORDERS IN ASSOCIATION WITH RHEUMATIC AUTOIMMUNE DISEASES IN CHILDHOOD
}

Liana Soido Teixeira e Silva (EPM-UNIFESP, São Paulo, SP, Brasil), Aline Maria de Oliveira Rocha (EPMUNIFESP, São Paulo, SP, Brasil), Annelyse de Araújo Pereira (EPM-UNIFESP, São Paulo, SP, Brasil), Jade Dib Fernandez (EPM-UNIFESP, São Paulo, SP, Brasil), Maria Teresa Terreri (EPM-UNIFESP, São Paulo, SP, Brasil)

\section{BACKGROUND}

Neuromyelitis optica spectrum disorders (NMOSD) is a severe inflammatory and demyelinating syndrome of the central nervous system classically characterized by attacks of optic neuritis and acute myelitis, with diverse neurological manifestations and possible association with other autoimmune disorders.

\section{CASE REPORT}

We present two cases of NMOSD manifested in patients with different autoimmune rheumatic diseases. A 15-year-old girl diagnosed previously with juvenile dermatomyositis. Two years later she presented an episode of optic neuritis which has improved after 5 days of methylprednisolone pulse therapy. After 4 months, she was admitted to the emergency room with complete spinal cord syndrome after a pharyngotonsillitis. Magnetic resonance imaging of neuroaxis showed longitudinal hyperintense lesion in the upper cervical, thoracic and lumbar region (Figure 1). NMOSD was diagnosed and anti-aquaporin-4 antibody showed to be positive. She received 5 days of methylprednisolone pulse therapy with minimal improvement followed by 5 plasmapheresis sessions performed every other day with significant recovery. Maintenance was initiated with rituximab and azathioprine. The second case was a 15-year-old girl without previous diagnosis, but a positive antinuclear antibody (homogeneous nuclear pattern 1:1280). Her monozygotic twin had systemic lupus erythematosus. She was admitted to the emergency room with 7 days of fever, headache, dizziness, vomiting, diplopia, visual blurring, lateral nystagmus and ataxic gait. Complementary exams showed anemia, lymphopenia, elevated erythrocyte sedimentation rate, altered urine protein-creatinine ratio, hematuria and direct positive Coombs test. She evolved quickly with brainstem and spinal cord syndrome. Magnetic resonance imaging of neuroaxis evidenced hypersignal foci in T2, affecting spinal cord in C6-T1 and T4-T7 levels (Figure 2). She was diagnosed with systemic lupus erythematosus and NMOSD. Anti-aquaporin-4 antibody was inconclusive. She received 5 days of methylprednisolone pulse therapy without improvement followed by 5 plasmapheresis sessions performed every other day with good response in addition to cyclophosphamide and gammaglobulin infusions. Her maintenance drugs were rituximab and azathioprine.

\section{CONCLUSION}

Association of autoimmune diseases is already well described and, in both cases above, two distinct autoimmune rheumatic diseases have been correlated to the same autoimmune neurological syndrome, manifesting in different intensities. We need to be aware to the atypical signs of NMOSD which may indicate another associated autoimmune disease. 